\title{
Bugged concrete
}

A special combination sensor for $\mathrm{pH}$ and $\mathrm{Cl}^{-}$has been made by Chinese researchers with the specific purpose of embedding it in reinforced concrete (R.-G. Du, R.-G. Hu, R.-S. Huang \& C.-J. Lin Anal. Chem. doi:10.1021/ ac517139; 2006). The device is simple - consisting of an Ir/IrO ${ }_{2}$ electrode and an $\mathrm{Ag} / \mathrm{AgCl}$ electrode - and

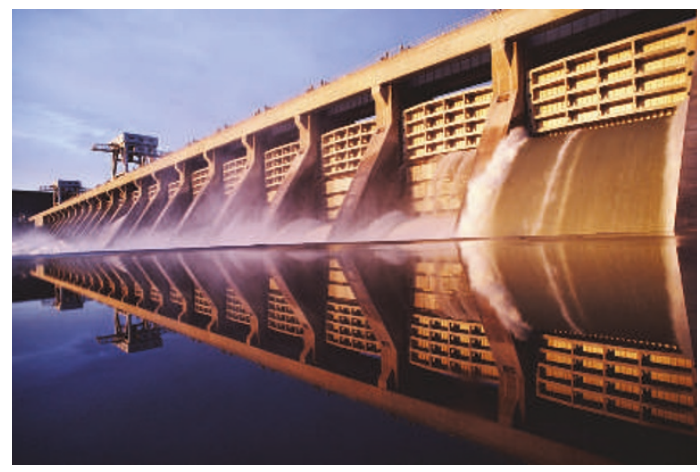
allows long-term studies of the two main parameters that cause deterioration and, ultimately, failure of reinforced concrete. Concrete is alkaline, and as long it remains at a high $\mathrm{pH}$, the interface between the concrete and the steel bars that reinforce it remains passive. However, problems arise when $\mathrm{CO}_{2}$, (which lowers the $\mathrm{pH}$ ) and $\mathrm{Cl}^{-}$(which accelerates steel corrosion) infiltrate through the concrete pores and reach the interface region. These structures can fail as a result of the steel rusting and unbinding from the concrete. So a sensor that simultaneously measures $\mathrm{pH}$ and $\mathrm{Cl}^{-}$concentration in situ will be more practical than the current multi-step procedures that destroy samples.

\section{A CLOSE LOOK AT DIAMOND}

After the discovery that the resistivity of diamond doped with boron drops to zero below a low but measurable temperature (1-10 Kelvin), it was proposed that this novel superconductor can be described, similarly to previously known superconductors, by the so called 'BCS theory', but in the dirty limit; that is, full of defects. Inspired by these recent events, Benjamin Sacépé and co-authors have examined the microscopic nature of boron-doped diamond at low temperature (B. Sacépé et al. Phys. Rev. Lett. 96, 097006; 2006). They used a scanning tunnelling microscope to reveal a clear energy gap between the carriers participating in the superconducting state and the other carriers, and to image vortices in magnetic field. Confirming what had been suggested, they could quantify both these observations by the BCS theory. The real surprise came when the vortices were examined more closely: they are arranged locally in a regular hexagonal lattice, and well-defined electronic states at energies within the gap exist inside the cores. Both these observations would not be expected in the dirty limit. This imaging experiment sheds new light on our understanding of diamond, demonstrating that what was considered a dirty superconductor is not that dirty after all.

\section{Playing hooky}

Researchers in Japan have unveiled an extremely simple approach for producing highly ordered macroscopic fibres of aligned nanotubes, in which the fibres can simply be hooked and pulled from an aggregate in solution (Y. Yamamoto et al. Adv. Mater. doi:10.1002/ adma.200502116; 2006). The fibres consist of amphiphilic hexa-peri-hexabenzocoronene (HBC) molecules, each containing thirteen fused benzene rings, which have already been shown to self-assemble into nanotubular objects made up of a helical array of $\pi$-stacked HBC units. Yamamoto et al. produce macroscopic fibres 5-30 $\mathrm{mm}$ long and $0.05-0.5 \mathrm{~mm}$ in diameter by ageing a solution of HBCs for three weeks to further grow the initially formed nanotubes into large entangled bundles. The authors then dip a glass hook into the solution to pull up and stretch the captured aggregate into macroscopic fibres. The authors find that, in contrast to simply casting the nanotube suspension on a substrate, the tubes in the pulled fibres are highly aligned. As a consequence, when the fibres are doped with iodine they exhibit highly anisotropic conduction - with more than an order of magnitude difference between the parallel and perpendicular measurements. Applications to nanometre-scale solenoids are envisaged.

\section{Polymers come full circle}

Polymers come in all shapes and sizes: linear, branched, hyperbranched, even dendritic. Less common are cyclic polymers, macromolecular chains with no beginning or end, whose properties are anticipated to be different from their acyclic counterparts. Although conceptually straightforward - the ends of a linear precursor are simply joined up — practical synthesis is not trivial. To favour cyclization over condensation, reactions are performed in dilute conditions, which demands an efficient coupling step. Researchers in the US have used 'click' chemistry — the copper-catalysed reaction between azides and alkynes — to solve this problem

\section{(B. A. Laurent \& S. M.} Grayson J. Am. Chem. Soc. Using atom transfer radical polymerization (ATRP), Laurent and Grayson synthesized linear polystyrene molecules bearing an alkyne group at one end and a bromine atom at the other. A simple chemical transformation for an azide group and the ends of the chain can then be 'clicked' together to form a cyclic structure. Although the polymer rings in this report are relatively small, the narrow size distribution control offered by ATRP, combined with the efficiency of 'click' chemistry, offers an attractive approach to these elusive compounds. doi:10.1021/ja0585836; 2006). swaps the bromine atom

\section{What's in a fibre?}

The integration of semiconductor devices into the structure of optical fibres opens up the possibility of more-efficient conversion between electronic and photonic signals. Writing in Science, Pier Sazio and co-workers (P. J. A. Sazio et al. Science 311, 1583-1586; 2006) report a technique based on chemical vapour deposition, in which vaporized semiconductor material suspended in an inert gas is forced through micrometre-scale capillary holes in optical fibres (pictured, scale bar is 1 $\mu \mathrm{m})$. The success of this method stems from the high pressures used, which cause semiconductor material to be deposited evenly along the length of the fibre. Using this technique, Sazio et al. show that silica capillaries, $100 \mathrm{~nm}$ in diameter, can be filled with semiconductor nanotubes with an inner diameter of less than $10 \mathrm{~nm}$. To test the applicability of this fabrication method in the devices, the group have produced a fully operational field-effect transistor that is encased within a silica capillary $94 \mu \mathrm{m}$ in diameter. It is expected that this method will find uses in the production of sophisticated fibreencased three-dimensional optoelectronic devices. production of real electronic

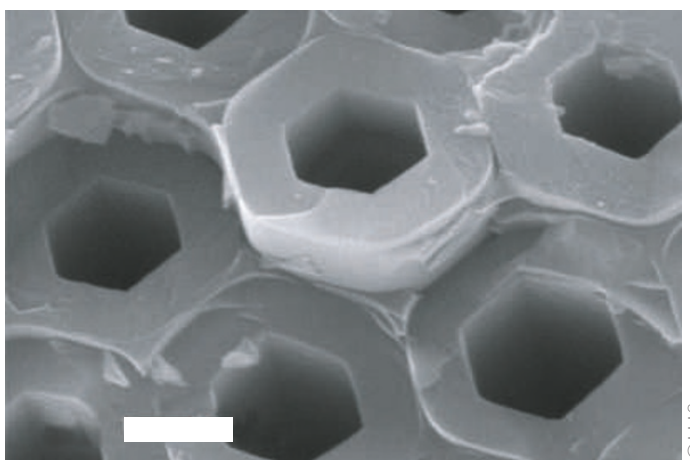

